\title{
Virtual Reality for Pain Control-Virtual or Real?
}

\author{
Parisa Gazerani
}

Department of Health Science and Technology, Aalborg University, Denmark

$\mathrm{V}$ irtual Reality (VR) technology creates a sense of immersion in a virtual environment analogous to the real world. VR has increasingly gained attention for pain management based on current evidence demonstrating its analgesic effects in certain experimental, acute and chronic pain conditions. VR-based interventions shift the users' attention towards active cognitive processing that can lead to higher pain threshold or tolerance. An understanding of neurobiological mechanisms underlying analgesic effects of VR will definitely facilitate optimal utility of this tool for pain control.

\section{Keywords}

Virtual Reality, Pain, Perception, Pain management, Analgesics

Disclosure: Parisa Gazerani has nothing to disclose in relation to this article. This article is a short opinion piece and has not been submitted to external peer reviewers. A member of the editorial board reviewed the editorial before publication. No funding was received for the publication of this article.

open Access: This article is published under the Creative Commons Attribution Noncommercial License, which permits any noncommercial use, distribution, adaptation, and reproduction provided the original author(s) and source are given appropriate credit.

Received: June 6, 2016

Published Online: August 30, 2016

Citation: US Neurology, 2016;12(2):82-3

Corresponding Author: Parisa Gazerani, Frederik Bajers Vej 7A2-A2-208, 9220 Aalborg East, Denmark.

E: gazerani@hst.aau.dk
Chronic pain affects millions of people around the globe. Current consensus considers chronic pain as a disease that arises from maladaptive changes within the central nervous system. Chronic pain poses a huge impact on affected individuals, societies, and health care systems. Current available medications or other treatment strategies are still far from providing an optimal, safe and adequate pain management for majority of patients. Hence, finding effective analgesic approaches would yield worldwide benefits. ${ }^{1}$ Novel technology-based interventions have been presented for chronic pain relief to address some limitations of pharmacology-based approaches. Those include but are not limited to neuromodulation, transcranial magnetic stimulation, and virtual/augmented reality (AR). Virtual reality (VR) offers digital recreation of a real life setting, while AR delivers virtual elements as an overlay to the real world. Both aim at creating sensory illusions. VR promotes behavioral engagement in a virtual environment analogous to the real world. AR, however, augments the real world by the addition of digital information. The two principal strategies employed are distraction and feedback. Distraction uses active cognitive processing to shift the user's attention and has been shown to increase pain threshold or tolerance. Feedback-based strategies are mainly used for phantom limb pain or complex regional pain syndrome..$^{2-5}$

Based on a recent meta-analysis of 14 controlled studies, VR-induced distraction has been indicated highly effective in alleviating pain. ${ }^{6}$ This analysis found a mean weighted effect size of 0.90 for VR distraction but it was not possible to draw any conclusion of VR effectiveness for acute versus chronic pain conditions. From included studies, seven were in experimental pain and seven in clinical pain. Between-group analysis indicated that VR was more effective in experimental pain compared with clinical pain. ${ }^{6}$ Another review, has found strong overall evidence for immediate and short-term pain reduction, and a moderate evidence for the reduction of pain and functional impairment after application of immersive VR. This review applied rapid evidence assessment strategy on 17 studies that consisted of five randomized clinical trials, six randomized crossover trials, two case series, and four single-patient case studies.? An extensive systematic review has also examined the effectiveness of VR therapy (immersive versus non-immersive) for pain management in children and adults. ${ }^{8}$ Non-immersive VR environment refers to the least interactive implementation of VR techniques (e.g. 2D interaction devices), while immersive VR environments (e.g. 3D immersive environments) provide highest interactive $V R$, in which subjects are fully immersed and interact with the VR environment. This review ${ }^{8}$ has provided the level of evidence of VR effectiveness for pain based on the Sackett criteria, where level 1a evidence (strong) was found for the effectiveness of immersive VR therapy in reducing acute pain; level $2 a$ evidence (limited) for reducing chronic pain and non-immersive VR for reducing acute pain, and level 5 (no evidence) for effectiveness of non-immersive VR for chronic pain. For children, there are no experimental studies to investigate the effectiveness of either immersive or non-immersive VR (level 5); however, level 2a evidence (limited) was found suggesting an advantage of immersive and non-immersive VR in reducing acute pain. ${ }^{8}$ Overall, it is evident that an immersive virtual 
environment has resulted in lower subjective pain ratings compared with watching a movie or playing games without VR. ${ }^{8}$

While existing evidence is promising for analgesic potentials of VR in acute painful conditions such as experimental pain models, burn injuries and dental procedures, only limited evidence is available in support of positive effects of VR in several chronic pain conditions. ${ }^{3,9,10} \mathrm{~A}$ recent randomized, controlled clinical study has investigated analgesic effect of Cryoslide (a VR game design, Pain Studies Lab, Simon Fraser University, School of Interactive Arts \& Technology, Canada) on patients with chronic pain recruited from a complex pain clinic in Vancouver." The study results demonstrated that pain intensity for chronic pain patients was reduced during the VR session. The authors proposed that the analgesic effect of Cryoslide is most likely based on a combination of effects including immersion, interaction, emotional engagement, cognitive distraction tasks, and transported presence experience. ${ }^{11}$ Maximizing sensory immersion and sense of presence (feeling of being in the virtual environment) in VR users have been shown to elevate VR analgesic properties. It has also been proposed that both the content of VR simulation, such as real life-like sensorimotor illusion, which allows optimal immersion, and VR's high level of interactivity, such as body movement might be considered for effective pain relief or rehabilitation protocols applied in pain management. VR systems (e.g. head-mounted devices) supplied with multi-sensory contents including vision, auditory, smell, taste and touch could potentially create an advanced level of immersion that would eventually enhance the analgesic effectiveness of VR.

A diverse range of VR system designs exist that are moving towards less expensive and more user-friendly versions (e.g. head-mounted Oculus Rift, Oculus VR, California, United States). VR approach has already entered into some hospital settings and will eventually be applied more routinely in clinical research and practice. The new devices are light, simple to set up, with short reaction time to help in preventing symptoms of simulation sickness, or cybersickness, which is a form of motion sickness with blurred vision, headaches, imbalance, nausea, and vomiting. Symptoms can occur during VR application or persist for hours after termination of VR. Simulation sickness seems to be understudied and there is no clear explanation on why it occurs, although some theories exist. For example, it is proposed to occur due to conflicts between visual, vestibular and proprioceptive perception. Considering these consequences of VR, it might not be a suitable choice for certain pain patients. Some researchers aim at modeling the sickness to predict the degree of sickness that might be expected from VR application.

It is yet not well known if VR could contribute to relief of anxiety or other comorbidities often accompanying chronic pain conditions. In addition, similar to pharmacological effect of a given analgesic drug, individual responsiveness to VR may exist. This technique may not be equally effective for everyone or all ages. Long term application of VR for chronic pain conditions may also carry some unwanted effects or challenges that have not been known yet, as most of the available data come from either acute experimental setting or short term investigations at clinic. It is also not known which type of chronic pain would respond better to VR and if there would be pain elevation after VR. There is so far one report available demonstrating that VR could increase pain. ${ }^{12}$

It seems reasonable to consider VR as a potentially valuable tool for chronic pain management. However, lack of sufficient evidence and potential challenges logically call for more efforts to obtain a better understanding of VR both in design and application for an optimal use in chronic pain management. Future studies may consider investigating the effects of age, gender, race and level of disability-functionality or medical status; identifying different factors that can affect analgesic effect of VR including quality, content, form and level or complexity; and also underpinning VR mechanisms at psychological and neurological levels. $\square$
1. Global Burden of Disease Study C, Global, regional, and national incidence, prevalence, and years lived with disability for 301 acute and chronic diseases and injuries in 188 countries, 1990-2013: a systematic analysis for the Global Burden of Disease Study 2013 systematic analysis for the
Lancet, 2015:386:743-800.

2. Zhang S, Seymour B, Technology for chronic pain, Curr Biol, 2014;24:R930-5

3. Li A, Montano Z, Chen VJ, Gold JI, Virtual reality and pain management: current trends and future directions, Pain Manag, 2011;1:147-57.

4. Mahrer NE, Gold JI, The use of virtual reality for pain control: a review, Curr Pain Headache Rep, 2009;13:100-9.

5. Malloy KM, Milling LS, The effectiveness of virtual reality distraction for pain reduction: a systematic review, Clin Psychol Rev, 2010;30:1011-8

6. Kenney MP, Milling LS, The Effectiveness of Virtual Reality Distraction for Reducing Pain: A Meta-Analysis, Psychology of Consciousness: Theory, Research, and Practice, 2016.

7. Garrett B, Taverner T, Masinde W, et al., A rapid evidence assessment of immersive virtual reality as an adjunct therapy in acute pain management in clinical practice, Clin J Pain, 2014;30:1089-98.

8. Shahrbanian S, Ma X, Aghaei N, et al., Use of virtual reality (immersive vs. non immersive) for pain management in children and adults: A systematic review of evidence from randomized controlled trials, Euro J Exp Bio, 2012;2:1408-22.
9. Wiederhold BK, Gao K, Sulea C, Wiederhold MD, Virtual reality as a distraction technique in chronic pain patients, Cyberpsychol Behav Soc Netw, 2014;17:346-52.

10. Shiri S, Feintuch U, Weiss N, et al., A virtual reality system combined with biofeedback for treating pediatric chronic headache--a pilot study, Pain Med, 2013;14:621-7.

11. Jin W, Choo A, Gromala D, et al., A Virtual Reality Game for Chronic Pain Management: A Randomized, Controlled Clinical Study, Stud Health Technol Inform, 2016;220:154-60.

12. Konstantatos $A H$, Angliss $M$, Costello $\mathrm{V}$, et al., Predicting the effectiveness of virtual reality relaxation on pain and anxiety when added to PCA morphine in patients having burns dressings changes, Burns, 2009;35:491-9. 